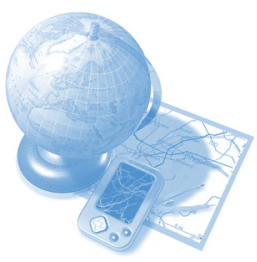

Gareth J Price
Department of Chemistry
University of Bath
Claverton Down
BATH BA2 7AY
chsgjp@bath.ac.uk

Our aim was to use ICT to enhance student learning by adding to our traditional teaching methods, not by replacing them.

\section{Computer aided assessment and feedback - can we enhance students' early experience at University?}

\author{
Abstract \\ This project, funded by LTSN Physical Sciences (Learning and Teaching Support \\ Network - now superseded by the Higher Education Academy Physical Sciences Centre) \\ and FAST (Formative Assessment in Science Teaching) was primarily concerned with \\ the production and evaluation of computer assisted assessment (CAA) materials to \\ support students in the first year of chemistry programmes. A number of short \\ assessment packages, designed to offer students formative feedback, have been written \\ and incorporated into WebCT. Questions were chosen to exemplify a range of styles and \\ were made available to students over the University computer network. The most \\ important aspect of the work was the feedback offered to students within the quizzes, \\ which was written in conjunction with undergraduate students to ensure its usefulness.
}

The effectiveness of the approach was evaluated by asking students to complete a questionnaire and by targeted interviews. The vast majority of the cohort (> 80\%) used the quizzes, most to gain formative feedback and some as a revision aid prior to end-ofunit examinations. This communication will summarise our findings and highlight some of the advantages and drawbacks in using electronic feedback.

Although there was a very significant set-up time involved as well as an on-going need for student support in using the packages, student reaction was positive and examination performance was enhanced over previous years. Although firm conclusions cannot be drawn from one year's data, these results together with the very positive reaction from the students encourage us to further develop the approach.

\section{Background and context}

Providing feedback to students on their work is vitally important if they are to succeed in their studies and particularly if they are to become independent learners. However, it can be extremely time consuming and demanding on staff to continually provide useful, individual feedback to students. Using electronic delivery has been suggested as having considerable potential in this area for enhancing student learning. This paper ${ }^{1}$ describes some work carried out in 2004 to design, implement and evaluate a virtual learning environment (VLE) based system for delivering formative feedback to first year students.

At Bath, a number of colleagues had some limited experience with using electronic question banks, of which there are many published examples, and electronic assessment packages such as QuestionMark Perception for both formative and summative assessments. However, we had not been able to take a coordinated approach and were concerned that some students were not fully engaging with our programme of workshops and tutorials and so were not receiving useful formative feedback until end-of-semester examinations. By this time it was often too late to fill gaps in knowledge or to correct misunderstandings since the teaching programme (which builds on this work) moves on at an increased pace. We were anxious to overcome this while trying not to 'spoon-feed' students; we wanted to develop a method that would enhance feedback and encourage them to take responsibility for their own learning during the early stages of their university careers.

The University was trialling the use of a VLE (in our case WebCT) and so we were keen to investigate whether this could help us. All first year students (apart from those at home - none in this cohort) live in University accommodation that has network connectivity which allows ready access to computer aided learning (CAL) materials to students who have a computer. The University also has a Learning Centre with $>450$ networked PCs which is open $24 \mathrm{hr}$ a day. It therefore seemed to us that CAA would potentially allow ready access to feedback. 
We were fortunate enough to obtain funding from LTSN Physical Sciences (now part of the Higher Education Academy Subject Centre Network) for a development project and for a case study as part of the FAST (Formative Assessment in Science Teaching) project ${ }^{2}$. The latter, discussed elsewhere in this journal, aims to develop successful approaches to formative assessment which enhance student learning. To quote from their project rationale ${ }^{2}$ :

"There is an urgent need to develop new and effective approaches to formative assessment which are cost-effective and which:

- Capture students' time and attention

- Generate appropriate kinds of learning activity

- Provide regular and timely feedback which has an impact on student learning"
The model adopted was to design and to implement, using a VLE, a series of self-assessment quizzes which students could try outside the formal teaching programme. A bank of questions would be developed covering a range of topics commonly found in first-year chemistry courses. In addition, a small number of work packages were to be written so that, in case of difficulty, students would have access to revision exercises. For example, if students consistently provided wrong answers to questions, they could work through a short, focused presentation on a topic (eg Valence Shell Electron Pair Repulsion - VSEPR) to remind them of the basic principles and then attempt some further assessment questions. The final, and most important, stage of the project was to evaluate in detail the effects (if any) that using the assessments had on student performance.

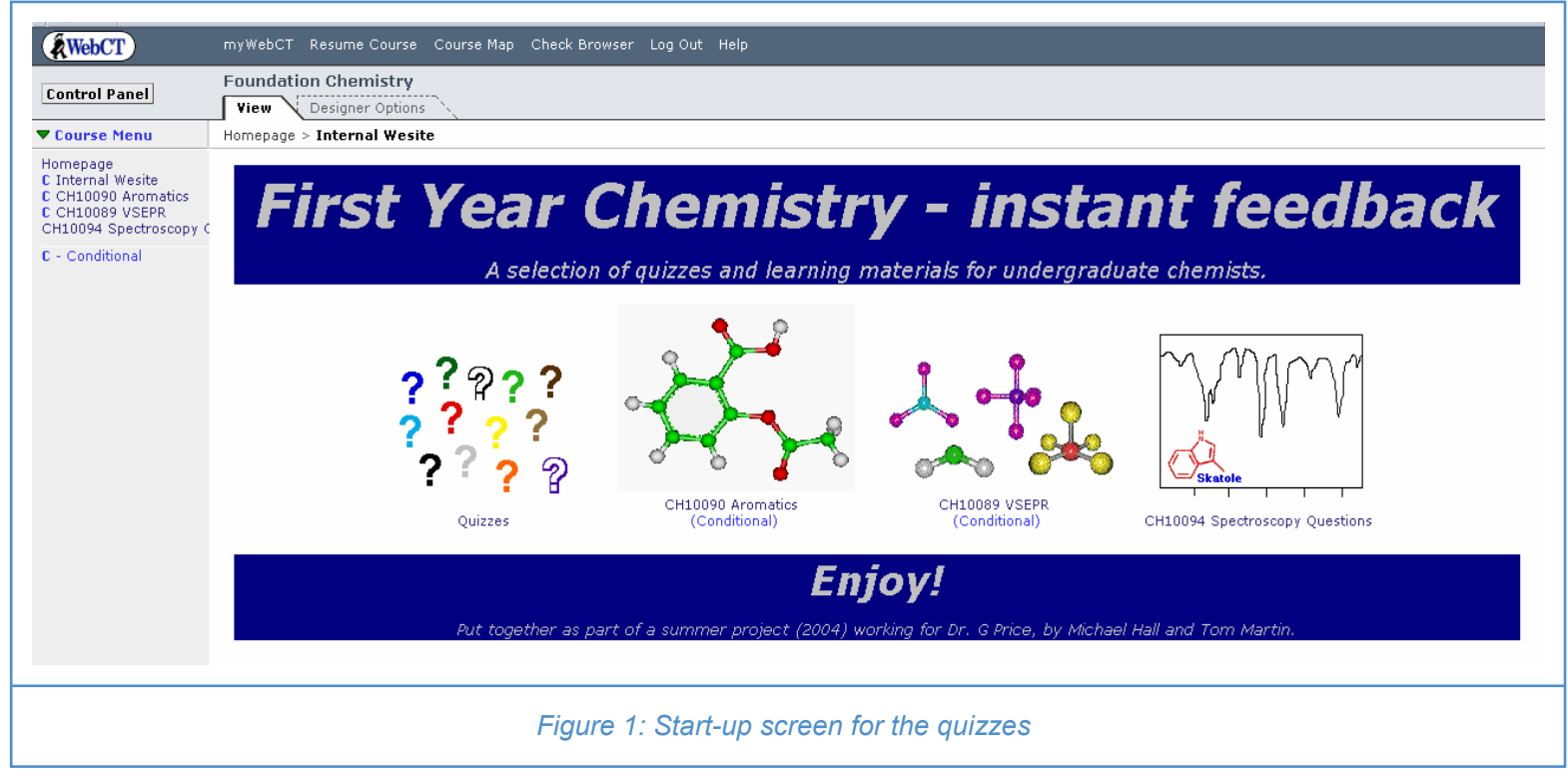

We took this as a framework on which to base our work.

The target audience was our Year 1, Semester 1 units in Chemistry covering a mix of Inorganic, Organic and Physical chemistry, ie the first units studied on arrival at University for BSc and MChem programmes in Chemistry or Natural Sciences. The student cohort consists mainly of school leavers with A-level qualifications although there are a number with International Baccalaureate qualifications together with a small number progressing from Foundation courses or from a GNVQ route. Chemistry teaching at Bath is based around a traditional lecture format (ca. 6 per week) supplemented by problem classes ( 2 per week) and small-group tutorials ( 1 per week) with 5-6 students. Prior to this project, most formative feedback was delivered during tutorial sessions.

The outcomes of the work were designed to be:

- a bank of questions suitable to provide formative assessment and feedback to students in the early stages of their chemistry course

- a number of revision and feedback packages

- an evaluation of the contribution made to students' initial learning experience

\section{Methodology and Implementation}

Gibbs and Simpson ${ }^{3}$ have summarised a series of conditions which should be satisfied if students are to receive useful feedback on their work. Our project was defined to meet a number of these conditions. One is that: "Feedback is understandable to students, given their sophistication". In order to ensure that this was the case in our work, undergraduate students (between years 1 and 2 of the MChem degree at Bath) were employed over Summer 2004 to develop the packages and advise on topics to be covered and on the level and language of the questions and feedback. This meant that all the questions and feedback would be at an appropriate level. It also meant that the topics chosen were those of concern to students and not simply those that academic staff 'felt' were difficult.

The front page from the WebCT package - dubbed by the students instant feedback' is shown in Figure 1. It provides access into the question banks ('Quizzes') and to three of the revision work packages that were written.

For each section (eg $2-5$ lectures) of material covered in lectures, a short multiple choice quiz (mcq) was written and mounted into WebCT. The aim was to allow students to test 
their 'basic' understanding of the fundamentals of the material as well as to give them a chance to find out whether they could apply this knowledge. The questions were based on some previously used at Bath, on textbook sources and on openly available internet sources. The students also wrote additional questions where needed. The staff member(s) teaching the units 'vetted' the questions for their suitability.

Question $5:(1$ point $)$

What mechanism is shown below?

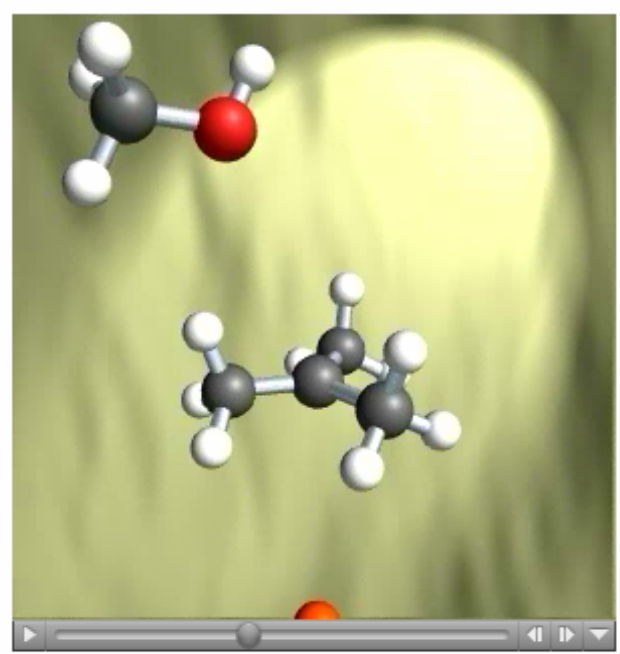

a. E1

b. $S_{N} 1$

c. $\mathrm{SN}^{2}$

d. E2

Save answer

Figure 2: Example question type

Some 20+ quizzes containing 8-15 questions each were developed. Questions were chosen to exemplify a range of styles covering basic subject knowledge, the ability to use and apply concepts developed during the lectures as well as, where appropriate, background maths and physics. One advantage of using a computer over a paper based system is that some questions were designed around animations to enhance students' understanding of eg reaction mechanisms and reinforce ideas of 'where atoms go' during reactions.

The quizzes were made available via the VLE on the University computer network and students 'encouraged' to use it during their studies in order to monitor their progress; it was not compulsory. However, an added incentive was that most of the 'past paper questions' from the unseen examination comprising part of the summative assessment for the units were included in the quizzes.

Individual quizzes were released in the VLE as the material was covered in lectures. In this way, students were able to assess their understanding immediately rather than having to wait for tutorials. Timeliness of feedback is another of the conditions for effectiveness suggested by Gibbs and Simpson.
So far, this scheme could be implemented simply by assigning reading and problems from a textbook. What is the advantage of using a VLE?

The main advantage offered is that constructive, formative feedback could be built in and made instantly available to the student. At the most basic level, students could use the test scores as an indication of their progress. However, we were careful to stress that students should use the quizzes to help develop a more independent style of learning and not simply focus on obtaining marks.

Simply telling students whether they had answered questions correctly or not would be of limited value. Into each question was therefore built some constructive feedback - drafted by staff but vetted and approved by the undergraduate students so that it is at an appropriate level to the user. Even if the question was answered correctly, feedback was given to enhance the learning (eg "Well done - you obviously remembered the correct units for the gas constant, R"), to reinforce good habits and to provide reassurance for less confident students that they really do understand the material involved. Incorrect answers were met with hints as to where students might have gone wrong. (eg "Have you considered the units of the gas constant?", "Think about how many joules are in a kilojoule" or "What does the ' 1 ' in ' $\mathrm{SN}_{1}$ ' mean?"). In this way, students were not simply fed the answer but forced to think about why they were not correct in the first attempt. In the event that they were completely unable to answer a question, students were directed to where they could get help. They were also encouraged to use the question as a basis for discussions during tutorials. Some of the question types are illustrated in Figures 2-4.

In an attempt to enhance the feedback given, some short 'revision packages' were written to be used when students needed further support on a topic. The principle that we tried to adopt follows the flow chart in Figure 5

This aspect of our work is at a very preliminary stage but represents an extension to how VLEs could be developed. In the main, students are currently directed to appropriate sections in lecturers' notes, text books or internet and other sources as appropriate. However, in one or two cases, we have prepared and incorporated short, highly focused revision packages which students can work through before returning to the self-assessment quiz.

Some technical problems encountered in incorporating material into WebCT meant that some clips could not be integrated with the quizzes as seamlessly as we had hoped. Nonetheless, student feedback was positive and it is hoped that further development of VLE interfaces as well as appropriate local implementation may allow much more effective use.

\section{Evaluation of the project}

The packages were used in Semester 1 and early stages of Semester 2 of 2004-2005. A full evaluation has been prepared $^{1}$ and will be summarised here. 
An initial evaluation of whether our approach was effective comes from a comparison of the unit results and the number of students who withdrew from the course during Semester 1 compared with previous years. During the study, only 1 student withdrew from the course before the Easter vacation compared with 6 in the previous session.
The primary evaluation of the project involved asking students to fill in a questionnaire together with some targeted interviews. The questionnaire was circulated after students had received their Semester 1 results, allowing time for students to reflect on their use of the quizzes.

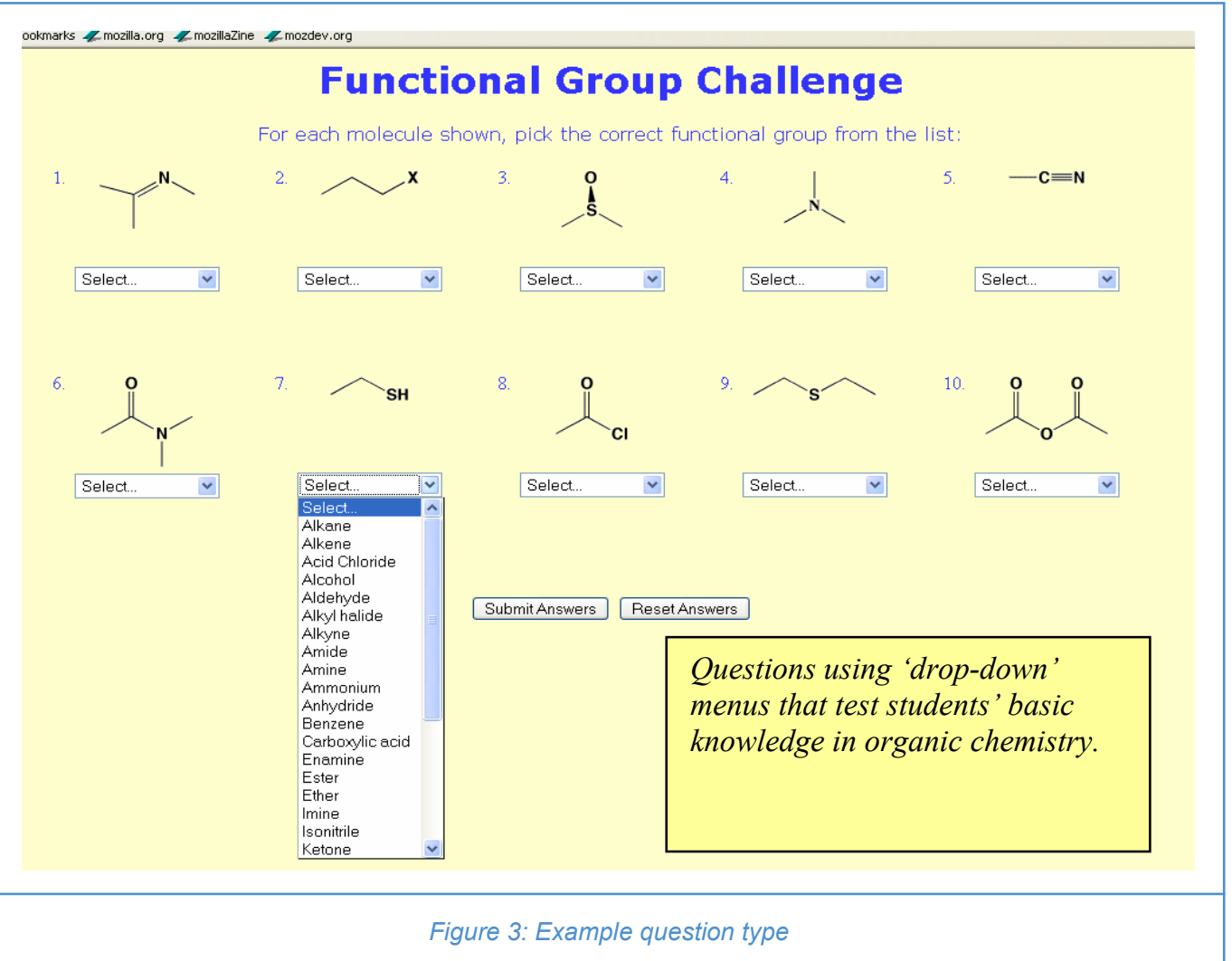

From a cohort of 115 students, 98 returned questionnaires, a response rate of $85 \%$. Of those who returned questionnaires, we were pleased to see that over $80 \%$ had used to the system to at least some extent. Given the well known cynicism of some students (the "it doesn't count so I won't bother" syndrome) this was satisfying.

The students who did not use the packages gave a number of reasons ranging from "Didn't have the time, kept forgetting." and "Didn't think it would be worthwhile" to those who tried but had technical problems. This is well illustrated by a comment "Attempted to use them but became frustrated with system's inability to handle 99\% correct answers. eg $99 \mathrm{kJmol}^{-1}$ was right but 99 (space) $\mathrm{kJmol}^{-1}$ was wrong". The latter comment clearly has implications for question design although it seems that this student was focusing

In terms of the summative assessment of the unit, there was a distinct improvement in performance for this session. The assessment comprised a piece of problem-based coursework done mid-way through the semester together with a multiple choice question examination and a problem based examination held at the end of the semester.

The cohort showed a significant improvement over the previous year with the average mark moving from 56.7 (sd = $13.4)$ to $65.2(s d=10.6)$. For each individual component, an improvement was shown with a pronounced (perhaps not unexpectedly) increase in the mcq examination where the average moved from 53.1 to 60.1 .

Of course this is at best a crude evaluation of the effectiveness of our approach. Many other factors affect performance and withdrawal rates. The average A-level entry grades were a little higher for the later cohort and this may account for some of the improvement. Part of the increased performance may simply have come from students' increased familiarity with question styles. However, we can at least conclude that the introduction of enhanced feedback has not harmed summative performance. more on getting the mark than acknowledging that they had obtained the right answer as an aid to learning! There were some interesting comments in terms of pedagogic factors including "I don't find computer learning particularly useful. I tend to remember things by rote if I use quizzes, instead of learning and understanding"; "Preferred to revise using books and notes with past papers, rather than using the computer, I don't really feel that MCQs are my favourite way to learn, I often feel extremely unmotivated to do them"; "I did not feel that the quizzes would help me, as they are not the style of revision that I know helps me the most" and "I would rather learn using a pen and paper!".

Given that current students are highly computer literate and we are sometimes told, regard traditional teaching as oldfashioned, We were surprised at these comments, albeit that they were small in number. The responses were anonymised so that it is not possible to correlate use of the system with final assessment marks.

Of the 80 students who did use the system, $65 \%$ used them for formative feedback during the semester, the others using them simply as a revision tool in the run up to examinations. Of the former group, about half used all the quizzes and of the rest, the preference was to use the quizzes for units that were found difficult rather than those in which the students were most interested. The majority of students felt that using the 
quizzes had helped them to learn the material covered in the units. While anecdotal in nature this, along with the improvement in examination performance, suggests that this was true.
- All students would use it after each lecture segment

- Tutorials could be better focused on student needs, improving their learning

- Fewer visits to staff with trivial problems would be needed leading to more effective use of staff time in dealing with problems

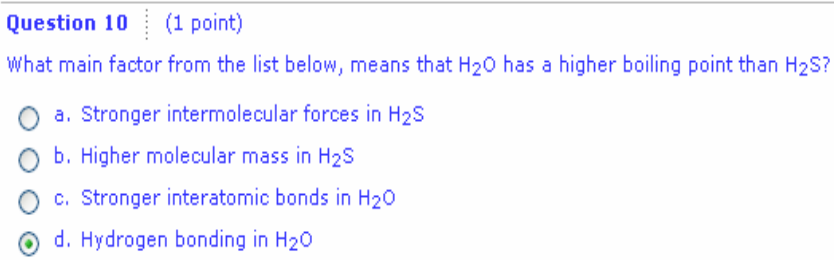

Questions that test students' ability to explain observations.

\section{Save answer}

Question $11 \quad$ (1 point)

Ethanol $\left(\mathrm{C}_{2} \mathrm{H}_{5} \mathrm{OH}\right)$ and dimethyl ether $\left(\mathrm{CH}_{3} \mathrm{OCH}\right.$ ) have the same molar mass. Which has a higher boiling point? (Hint: think about what type of intermolecular forces occur in each species).
() a. Ethanol
b. Dimethyl ether

Questions that test students' ability to apply a concept.

\section{Save answer}

Question 12 (1 point)

Which two statements can be deduced from the Lennard-Jones expression?

$U(r)=\left\{\frac{b}{r^{12}}-\frac{a}{r^{6}}\right\}$

a. At long range the repulsive forces are stronger than the attractive forces.

$\square$ b. That the attractive forces act over a longer range than the repulsive forces.

c. At long range the attractive forces are stronger than the repulsive forces,

d. That the repulsive forces act over a longer range than the attractive forces.

Questions that look at students' background mathematics.
Students though were neutral on whether the feedback had helped them to actually plan their study or whether use of the system helped in bridging the school-university transition.

There was a slight preference for the suggestion that using the packages helped to develop independent learning, although few students seemed to have used the feedback as a basis for seeking further help during tutorials. Only 10 students felt that the CAA approach was better than the traditional tutorials, even though it is more readily available. A larger proportion of the class used the feedback quizzes as an aid to revision for the final assessments. Of these 80 students, all but 9 used the quizzes to gauge how their revision was proceeding and the majority used them as a diagnostic tool to focus their revision and agreed that the feedback was helpful in learning the material. The ability to get answers at any time was, not surprisingly, regarded as an attractive feature. Again perhaps not surprisingly, students expressed strong preference for visiting tutors to get problems answered rather than simply using electronic means.

\section{Discussion and comments on good practice}

Overall, we were pleased with the student acceptance of the work and the apparent success of the project. When we designed the package, our hope was that:
- More effective revision with less need to visit staff during revision time

- Better performance in assessments

- Higher student satisfaction with Semester 1 studies.

So, what was the result? A high proportion of the students used the system to get formative feedback during the semester while a smaller group used it as a revision aid. Most students felt that using the quizzes had improved their overall performance and this is supported by the change in average marks, albeit for a single cohort.

The main unforeseen circumstance that we encountered was the comparative overloading of students in the first few weeks of their university careers. Although we had hoped that our feedback system would help in the school-university transition, it was hardly used in the first few weeks. Enquiries to students showed that many were overwhelmed by the number of new procedures, tasks, skills and general activities that take place in the first couple of weeks, both academically and socially. A second introductory session was held after 4-5 weeks of the semester and usage increased afterward. 
We underestimated the time commitment required to set up such a package of quizzes, even when using a commercial software product such as WebCT. We were pleased at the comparative lack of technical problems faced by students albeit that this was offset by the staff set-up time spent ensuring that things were robust.

The initial set-up time and technical support necessary for such a system should not be underestimated. Sourcing, devising and inputting the questions was time consuming (ca.

\section{Conclusions}

So, in terms of effective feedback, our system meets many of the criteria of Gibbs and Simpson. The packages are released in parallel with the lecture material so that it is relevant and gives students the opportunity to monitor their progress in time to seek assistance if necessary. The feedback given is 'instant' - available from a PC at any time. The quizzes were designed to cover both revision of background knowledge, basic understanding of new material and the ability to use and apply concepts so that a range of student skills was needed.

The feedback given should have been
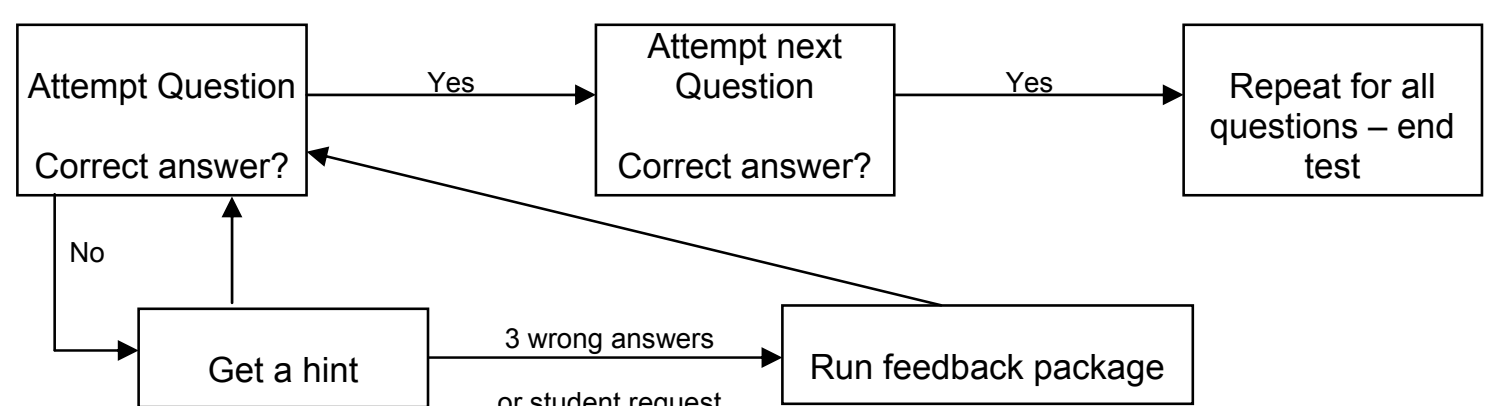

or student request at the appropriate level for students and we were continually careful to emphasise the diagnostic nature of the quizzes. The marks achieved were not important: the main aim was to allow students to monitor their progress and so help them to take responsibility for their own learning.

13 weeks for the summer students). Even though a commercial VLE was used, there were technical issues in its use in terms of student access, passwords etc.) and in working out how to include some question types (eg those with video clips or the interface with Powerpoint). Individual students also needed help with accessing and navigating the system so that some further support during the Semester was vital to the successful operation.

A number of technical issues cropped up during implementation of the VLE. Mainly, these are to do with local implementation but the major operational one concerns the way in which WebCT structures quizzes. The same problems were encountered when using QuestionMark Perception for similar purposes. Neither package is ideal for 'formative' assessments. In fact, the University of Bath recently made the decision to adopt Moodle as its VLE and so we are about to undertake the migration of the packages to this new system and this may overcome some of our problems.

Our aim was to use Information and Communication Technologies (ICT) to enhance student learning by adding to our traditional teaching methods, not by replacing them. In this we seem to have been successful, at least in terms of student acceptability. One telling comment which applies to CAL methods in general rather than specifically to this project was "I came to Bath because of the friendliness and approachability of staff - and then you send me away to work with a computer on my own". Clearly, we need to manage the introduction of CAL carefully if detrimental changes to our departmental ethos are not to occur.

\section{Acknowledgements}

We are grateful to the FAST project and the LTSN (now Higher Education Academy Physical Sciences Centre) for funding some of this work. In particular, Evelyn Brown was our 'FAST mentor' and gave us valuable help, particularly in the evaluation of our approaches.

In writing this paper I need to thank Mike Hall and Tom Martin, two undergraduate students who worked on the project and contributed many ideas of their own from the student point of view. Also, the input of other staff at Bath who contribute to $1^{\text {st }}$ year teaching is acknowledged.

\section{Notes and references}

1. This paper is based on the final report of the development project which is available at: $h t t p: / /$ www.physsci.heacademy.ac.uk/Resources/ DevelopmentProjects.aspx (accessed Jun 2006)

2. For details of the FAST project, see: $h t t p: / /$ www.open.ac.uk/science/fdt//overview.htm (accessed Jun 2006)

3. Gibbs G. and Simpson C., Conditions Under Which Assessment Supports Students' Learning, Learning and Teaching in Higher Education 1, 3-31 (2004-5) 